\title{
Understanding the Perspective of Behavioral Economics' Planning Fallacy in Improving Business Strategy for Kopi Bon
}

\author{
Fadhilla Sandra Adjie, Alvanov Z. Mansoor, Sony Rustiadi, and Ira Fachira
}

\section{ABSTRACT}

In seeking differentiation value, Small Medium Enterprises tend to have limitations which hinder their way in developing the business strategy. As an SME that has established its two-year business operation-Kopi Bon is being analyzed and investigated from the perspective of behavioral economics' Planning Fallacy-as it shows that the planning process is filled with distortion made from judgments as a result of behavioral biases. The study revealed how cognitive biases that involve planning fallacy as a supporting theory influences the company's decision-making process-where later could be affected to the decision making in terms of operational activities which comprises of the quality of products and services, to the company's branding. Also, the study reveals how understanding planning fallacy would make improvement towards business strategy to survive within the pandemic of COVID-19.

Keywords: Planning Fallacy, Small Medium Enterprises, Decision Making, Cognitive Biases, COVID-19.

\author{
Submitted : May 04, 2021 \\ Published : May 25, 2021 \\ ISSN: $2507-1076$ \\ DOI: $10.24018 /$ ejbmr.2021.6.3.873 \\ Fadhilla Sandra Adjie * \\ Bandung Institute of Technology, \\ Bandung, West Java, Indonesia. \\ (e-mail: fadhilla_sandra ${ }^{@}$ sbm-itb.ac.id) \\ Alvanov Z. Mansoor \\ Bandung Institute of Technology, \\ Bandung, West Java, Indonesia. \\ (e-mail: alvanov.zpalanzani @ ${ }^{\text {sbm-itb.ac.id) }}$ \\ Sony Rustiadi \\ Bandung Institute of Technology, \\ Bandung, West Java, Indonesia. \\ Ira Fachira \\ Bandung Institute of Technology, \\ Bandung, West Java, Indonesia. \\ *Corresponding Author
}

\section{INTRODUCTION AND RESEARCH OBJECTIVES}

Along with the rise of Small Medium Enterprises until the end of 2020, there are various examples whether the businesses survive and significantly grow that later lets them to validate the business and scale up, or conversely decline the company. Furthermore, each of the businesses might have its different standards and parameters in meeting the customers' demands, however in terms of having a wellplanned and organized business planning — it is notable to understand that there are some potential distortions made from judgments. For instance, how can a business have made operational and management plans in order to gain more revenue after considering along with calculating project cost and timeline, but contrarily the project takes longer and cost more than expected. In addition, as an attempt in responding to this example of varied interference in business - we would like to have a deeper understanding by applying behavioral economics, particularly acknowledging the term of planning fallacy - the supporting theory to elaborate how cognitive bias occurs within our thinking process. This term might be helpful to reduce biases that affect organization performance and planning effectiveness.

\section{A. Statement of the Problem}

In the midst of Pandemic COVID-19, as a Small Medium Enterprise (SME) - Kopi Bon had to survive the business particularly in meeting customer's demands that are various in which also diverse and unpredictable. However, to make ends meet the business encountered difficulties in having a well-planned and organized business planning without any distortions that blurred the company's decision-making.

\section{B. Research Objectives}

The present study is an attempt to meet the following objectives, namely:

1. To study behavioral economics by using planning fallacy as a part of cognitive bias, also to bring out the familiarity for modern economics.

2. To recognize the business challenges in order to develop the company's strategies, also to reflect and figure out the current condition - being in the midst of a pandemic COVID-19 situation.

3. To develop an understanding and minimize planning fallacy which in return may improves Kopi Bon's business strategy.

\section{RESEARCH METHODOLOGY}

The study aims at improving Kopi Bon's decision making while acknowledging how impactful cognitive bias towards it, and how important it is to recognize also reduce planning fallacy within the business strategy process of making decisions. Since the research is targeted in observing the management team as a way to identify business' method in decision making, also to identify how impactful the 
management's decision towards the company's revenue sales — by involving customers as the respondent.

The research methodology of this study uses literature studies also using qualitative method to determine the empirical validity of the study above in attempt to integrate perspective and create broader horizon by involving several ways of collecting data with a touch of social interaction that gives ease to the observer, namely [1]:

\section{- Interview}

It is an essential component of qualitative research in collecting data, and also lets the participants have opportunity in discussing any challenges they had.

\section{- Focus Group or Group Interviews}

Focus Group will help the observer in using useful techniques for obtaining individuals' impressions and concerns about certain difficulties. The activity should be participated from 6 to 10 individuals and a trained moderator that shares participant's characteristics, demographic, or their interest that is relevant to be studied by the observer.

- Surveys

Asking numerous people to question their behaviors, options, and options. It is conducted to find and assess the relationships between the characteristics of respondents and their reported results. In providing the activity, it requires nine steps as follows:

1. General Objectives.

2. Specific Objectives.

3. Sample.

4. Questionnaire.

5. Fieldwork.

6. Content analysis.

7. Analysis Plan.

8. Tabulation.

9. Analysis and Reporting.

\section{EXTERNAL ANALYSIS}

\section{A. Cognitive Biases}

Human brain has two modes of thinking, intuitive that represented as System 1 in which works with associations feelings, intentions, etc. Then there is reflective that represented as System 2 wherein functions slowly, effortful, and deliberate - according to cognitive scientist [2]. Cognitive bias might be defined as a systematic error in thinking which affects one's decisions and judgments of people make-sometimes these biases are related to memory. A person's recollection of certain occasions might be biased for various reasons, which later lead to biased thinking and decision-making. Also, cognitive biases might be related to problems with attention-wherein is a limited resource, people have to be selective about what they pay attention to in the world around them [3]. This cognitive helps us in addressing four major problems, namely:

1. Confirmation Bias, where there is too much information to deal with - also our System 1 brain selects the information that we are most likely to consume.

2. Functional Fixed, where human attempt to make sense out of what they perceive, regardless of the information does not elaborate enough meaning.
3. Attentional Bias, where we need are narrowed; to think and also act fast in decision making.

4. Misinformation Effect, where we should remember information that was consumed beforehand.

In particular, Kahneman and Tversky argued that biases contribute to changing human behavior, wherein form the notion of incentives of nudges. Furthermore, Kahneman also elaborated that a person could not acknowledge his own biases, however in contrary he could easily spot and perceive others' errors [4]. In other words, he discovered that human is an irrational human being — as he explicated that our decisions are based on shortcuts or heuristics, in which are derived from precedent experiences that affect our decision making that later affects our behaviors as well.

\section{B. Planning Fallacy}

The brain can be deceptive in terms of rational decision making. As a part of the cognitive bias - Planning Fallacy came up with the notion where describes unrealistic plans and forecasts, or in other words where people tend to underestimate the time required to complete certain projects or complex jobs, even when they have considerable experience of past failures in an attempt to keep up with the likelihood of the project itself. The basic concept was first presented by Daniel Kahneman and Amos Tversky in 1979, who were formerly introduced the idea of cognitive biases, and their impact on decision making in 1974.

In a business industry, it has been found that more than $80 \%$ of start-up ventures fail to achieve initial market-share targets, as demonstrated by the cognitive scientist. For instance, we can refer to the real case of planning fallacy from Sydney Opera House. Its construction had unforeseen difficulties which delayed the whole construction that affected the lead time which takes a decade longer than planned beforehand. Also, the construction cost was projected for merely $\$ 7$ million, however surprisingly it had cost $\$ 102$ million by the time the construction was finished. Another following problem occurred to the Canadian Pacific Railway in 1871, wherein initially was planned to be completed in 1881. However up until 1885, let alone the railway was not completed, it charged more costs that reached $\$ 22.5$ million which could be fulfilled through the loans that was far from former prediction [5].

To explicate more about Planning Fallacy — Kahneman and Tversky in 1979 used a psychological mechanism that determines people's optimistic forecasts by using The Inside versus Outside view. The term analyzes on how people percept towards a planned project, where they tend to merely focus on the inside of their ability such as involving information's aspects of an internal point of view such as merely focuses on the duration of completion times while neglecting outside's view even if it is relevant to the project itself and how it fits to the set of related tasks [6].

\section{BUSINESS OVERVIEW AND ITS CHALLENGES}

Kopi Bon is a coffee store wherein does not merely sell beverages, however it differs itself from another coffee shop by providing creative event organizer independently when other brands do the similar way while collaborating with a creative organizer. The tickets sold from creative event 
organizer are converted to the food or beverages menu, as preferred by the customers. The strategy of providing creative event organizer also function as a company's marketing strategy to raise more income, alongside with the management's effort in a cost-efficient way.

After proceeding the business for two years, the management team analyzes the pattern of the company, the market, even behavior of their customers. However, due to the appearance of COVID-19 - Kopi Bon keeps surviving, while also adapting with various shifts that the pandemic undertook in which also led to the shifting of company's policies and decision making. As the new pattern could not be foreseen, nonetheless the management team needs to act fast and become very well adaptive.

Primarily, the reason why Kopi Bon became the subject of our analysis is based on its establishment for the last two years - also the business growth has not going significantly well within the business process after COVID-19 strikes. Furthermore, the current situation hit the business hard and made Kopi Bon to keep operating while optimizing its selling merely via online which affects the sales that decline. Hence, Kopi Bon before doing the company's forecast, the management should understand the term of planning fallacy when in making decisions does not based on delusional optimism which later caused bad planning, or even worse leads to bad investment for the financing.

\section{BUSINESS SOLUTIONS}

Furthermore, in the midst of COVID-19 - irrational behaviors might intensify as an impact of the pandemic's fallout. As a Small Medium Enterprise, Kopi Bon has to consider the importance of what the consumers are thinking and how they are reacting towards any company's arrangement — from marketing and sales policy, the quality of products and services, to the job policy for employees. Moreover, in order to decide what strategies suit best - is to understand how bias gets to people's thoughts and influence the thinking also their behavioral shifting [7].

Alongside with the explication of planning fallacy, the major substance of the theory is to understand that in order to predict the future - it is not significantly advised to rely on the past information which does not give any accurate forecasting. For instance, when $\mathrm{H} 1 \mathrm{~N} 1$ - the prior health crisis occurred in 2019 people did not seem to have panic buying in mind, as we see the fact that when COVID-19 initially transmit - it led to the shortage of health products such as hand sanitizer, health mask, and antiseptic. The examples given also indicate that cognitive bias constructed without any prior proclamation in any past events. Therefore, the management team needs to evaluate what are the strategies involved to tackle another crisis that also acknowledge biases which led the declining sales and revenue - also preparing the post-crisis situation to survive.

Kahneman, Lovallo and Sibony in 2011 had revealed a decision quality control by using a checklist within the research methodology process - through interview or focus groups. These developed tools help the management team to examine their decisions based on a 12-question checklist. Comprises into three categories; questions the decision makers should ask themselves, questions they should use to challenge the people proposing a course of action, and questions aimed at evaluating the proposal — as shown below.

TABLE I: DECISION QUALITY CONTROL: 12-QUESTION CHECKLIST

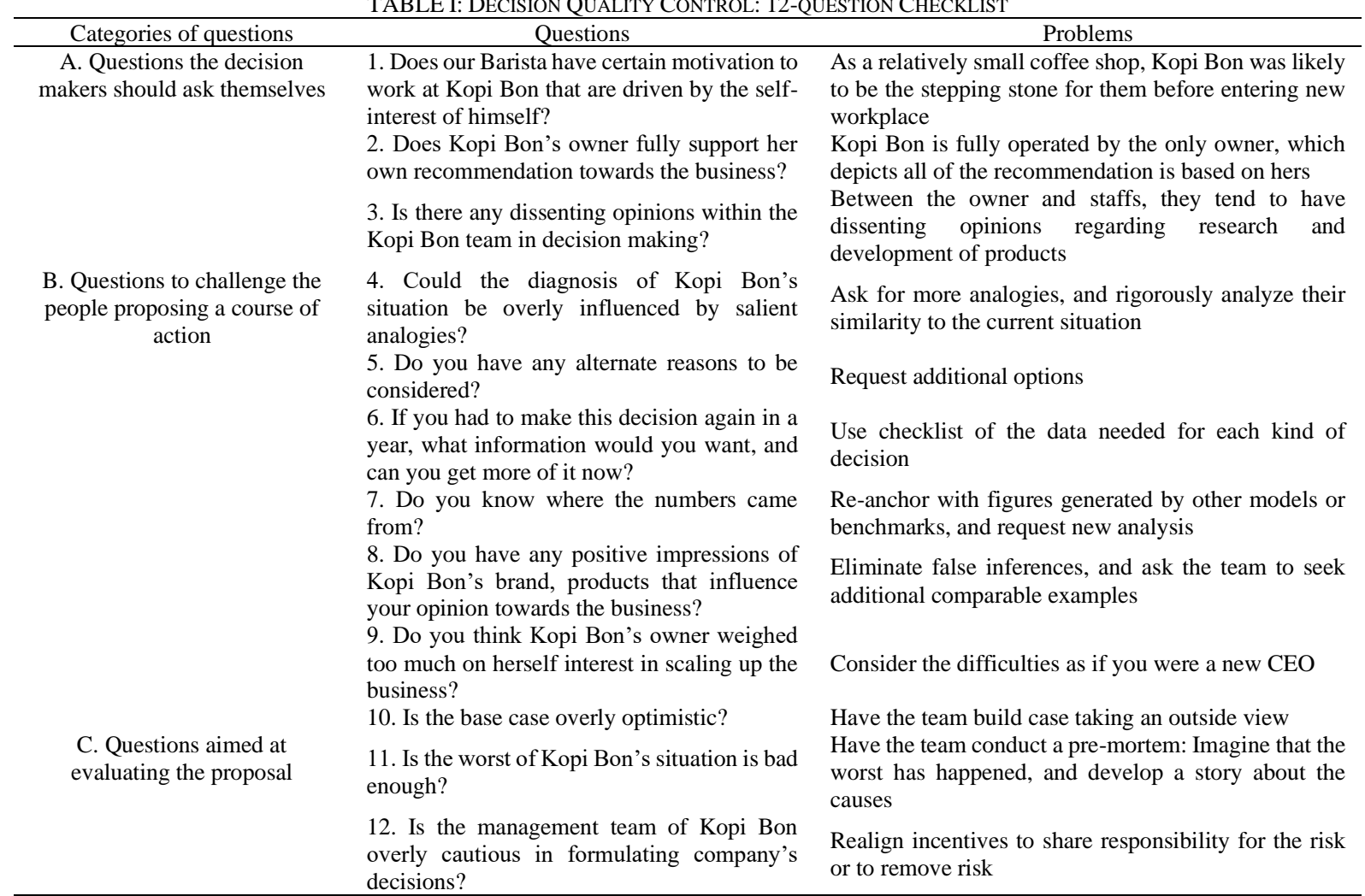


These 12-questions checklists [2] will be substantially helpful, particularly for those who are in management team, namely:

1. Understand the best time to use 12-questions checklist. In this term, Kopi Bon might improve its quality control of both products and services, also to decide where the capital expenditure might be wisely spent. According to the SelfEvaluation question above, alongside with all of the recommendation is based on the owner's decision, thus in order to get a more objective and diverse opinions - Kopi Bon could develop the flow of decision making by contributing more of its staffs.

2. Understand who should conduct the review, as it comprises the one who becomes the decision maker, and the team making recommendations. Hence, before going on to further research - Kopi Bon's management team needs to resolve and decide which team and who will make the recommendation, to debias the notions from the decision maker. In addition, besides the mere focus on the owner as decision maker, she considers alternative strategies from the Head Barista and Supervisor as the frontline of all the staffs that contribute as a mediator of the owner and with them.

3. As a decision maker - Kopi Bon's owner needs to have standardized regulation, which is important to go through the checklist completely, systematically, and routinely. According to this, Kopi Bon's management team needs to be prepared for systematic cultures - that might later be evaluated. In this particular situation during pandemic COVID-19, Kopi Bon optimizes and generate more sales through the online platforms. The standardized regulation could be beneficial in providing meticulous operational changes under fluctuating circumstances. Thus, even though the owner and staffs have dissenting opinions, through the means of standardized regulation - this would help to guide both stakeholders to stick with the business' procedures.

4. Evaluate the cost and benefits in each and every decision that are made. As every business needs to scrutinize this aspect, however the term might also be considered biased as it turns out that a company's management is having the problem of overoptimism and overconfidence that comes from internal judgment. Hence, Kopi Bon needs to involve more teams as a way to be the recommendation team and reduce bias. According to the Self-Evaluation questions, in order to reduce bias recommendation that merely based on the owner's, Kopi Bon should contribute more of its staffs in gathering varied perspectives to formulating recommendation. For instance, during pandemic staff who could not work according to the owner's work schedule might not get any monthly reward - as this considered as a benefit context. Conversely, when Barista is having a well-performed work ethics that also help owner to upsell Kopi Bon's products particularly during pandemic situation, the owner would give a bonus accordingly.

However, these four elements mentioned might be changed when the overall 12-question checklist is all answered by each and every stakeholder involved.

\section{CONCLUSION}

The study aimed to contribute to giving an understanding of how cognitive bias influence a company in terms of decision making, particularly under fluctuating circumstances of COVID-19. By contributing Kopi Bon as the object study, we analyze how an internal judgment of a company might be impactful towards the output such as sales revenue, marketing strategy. Through planning fallacy, it reveals how developing strategic plan are based on estimations of what might happen overtly or covertly. This fallacy should be reduced by the business owner who have the major responsibility to react on the occurrence of any kind of situation the company has. In addition, as a decision maker - the owner recognize planning fallacy helps to restructuring decision plans within the business, for instance she might be able to understand operational strategies that are not successful to be applied in the pre-pandemic situation and analyze which ones that is helpful in handling the business under pandemic situation.

Kopi Bon needs to reduce the effect of cognitive bias in its company, by implementing decision quality control checklist. Not merely using tools, Kopi Bon needs to evaluate its business sequentially and involve holacracy — an approach regardless of hierarchy within the management team in order to get a diverse perspective, so the cognitive bias could be reduced. In return, the business would be focused on the strategies set in the first place. Thus, the usage of Decision Quality Control (12-question checklist) as a tool might be applicable to maintain company's attempt in maximizing the organization's efficiency and attention by mapping out its strategies in a more objective way. Through the means of Decision Quality Control, Kopi Bon might be able to:

1. Improving quality control for both products and services that also contribute staffs in order to get a more objective and diverse opinions.

2. In decision making within the business, the involvement of another alternative recommendation from Supervisor and Head Barista as a mediator of the owner and staffs - is notable to be considered.

3. As a decision maker, Kopi Bon's owner needs to get used to a more standardized regulation, in order to get have an effective way of working and also providing meticulous operational changes under fluctuating circumstances such as during pandemic COVID-19.

4. Help to evaluate the cost and benefits in each and every decision that are made. Notably during pandemic that offers the uncertainty, hence the cost and benefit helps to assess which strategies that are costly and should be reduced, and which ones that are beneficial to be applied for the business longevity.

\section{REFERENCES}

[1] G. Marczyk, D. DeMatteo, and D. Festinger, Essentials of Research Design and Methodology, New Jersey: John Wiley \& Sons, Inc, 2005, pp. 153-154.

D. Kahneman, D Lovallo, and Sibony, O. (2011). Before You Make That Big Decision. Harvard Business Review, [Online]. Retrieved from: https://hbr.org/2011/06/the-big-idea-before-you-make-that-bigdecision.

[2] H. H Friedman (2017). Cognitive Biases that Interfere with Critical Thinking and Scientific Reasoning: A Course Module. Brooklyn College of the City University of New York. 
European Journal of Business and Management Research www.ejbmr.org

Yagoda, B. (2018). The Cognitive Biases Tricking Your Brain. Retrieved from: https://thetheatlantic.com/magazine/archive/2018/09/cognitivebias/565775/.

[3] The Decision Lab.com (2021), Planning fallacy - Biases \& Heuristics. Retrieved from: https://thedecisionlab.com/biases/planning fallacy/\#: :text=The\%20Sydney\%20Opera\%20House $\% 20$ is,complete $\% 20$ than $\% 20$ was\%20originally\%20planned.

[4] R. Buehler, D. Griffin, and J. Peetz. (2010). The Planning Fallacy: Cognitive, Motivational, and Social Origins, Journal of Advances in Experimental Social Psychology, 43 pp. 17.

[5] Kadence Report (2020). Cognitive Biases Explaining Irrational Behaviours Worldwide. Retrieved from https://kadence.com/cognitive-biases-explaining-irrationalbehaviours-worldwide/.

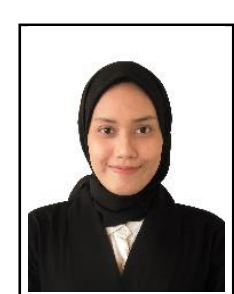

industries as well.

Fadhilla Sandra Adjie was born in February 27, 1996 in Bandung, Indonesia. Graduated from International Relations at Parahyangan Catholic University in 2017. She pursued her Master degree in Master of Business Administration at Bandung Insitute of Technology, Indonesia, and graduated in 2021, during her postgraduate life her interest in Business and Management, Consumer Psychology, and Behavioral Economics grows. Currently as an entrepreneur, she would like to deepen her career in academic world and business

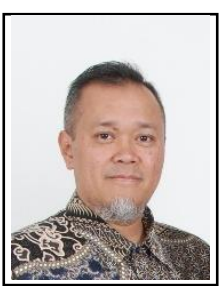

Alvanoz Z. Mansoor was born in November 27, 1974 in Bandung, Indonesia. He completed his doctoral program in Institute of Technology Bandung majoring in Art and Design studies in 2012 after graduated from Master of Management program, Telkom University in 2005. His expertise in research are cultural studies, media studies, visual storytelling, and creative business. Dr. Mansoor, is currently works as lecturer, researcher, boardgame designer, and creative business and visual storytelling consultant. Dr. Mansoor is eager to contribute to cross and multidiscipline studies and various creative projects. 